\title{
Observations of Coronal Mass Ejections with the Coronal Multichannel Polarimeter
}

\author{
H. Tian • S. Tomczyk $\cdot$ S.W. McIntosh $\cdot$ C. Bethge $\cdot$ \\ G. de Toma $\cdot$ S. Gibson
}

Received: 31 December 2012 / Accepted: 2 May 2013 / Published online: 5 June 2013

(C) The Author(s) 2013. This article is published with open access at Springerlink.com

\begin{abstract}
The Coronal Multichannel Polarimeter (CoMP) measures not only the polarization of coronal emission, but also the full radiance profiles of coronal emission lines. For the first time, CoMP observations provide high-cadence image sequences of the coronal line intensity, Doppler shift, and line width simultaneously over a large field of view. By studying the Doppler shift and line width we may explore more of the physical processes of the initiation and propagation of coronal mass ejections (CMEs). Here we identify a list of CMEs observed by CoMP and present the first results of these observations. Our preliminary analysis shows that CMEs are usually associated with greatly increased Doppler shift and enhanced line width. These new observations provide not only valuable information to constrain CME models and probe various processes during the initial propagation of CMEs in the low corona, but also offer a possible cost-effective and low-risk means of space-weather monitoring.
\end{abstract}

Keywords Active regions · Coronal mass ejections · Flares · Magnetic fields · Waves

\section{Introduction}

Coronal mass ejections (CMEs) are probably the most important sources of adverse spaceweather effects (e.g. Gosling et al., 1991; Gopalswamy et al., 2001; Wang et al., 2002;

Coronal Magnetometry

Guest Editors: S. Tomczyk, J. Zhang, and T.S. Bastian

Electronic supplementary material The online version of this article (doi:10.1007/s11207-013-0317-5) contains supplementary material, which is available to authorized users.

\footnotetext{
H. Tian

Harvard-Smithsonian Center for Astrophysics, Cambridge, MA, USA

e-mail: hui.tian@cfa.harvard.edu

S. Tomczyk $(\bowtie) \cdot$ S.W. McIntosh $\cdot$ C. Bethge $\cdot$ G. de Toma $\cdot$ S. Gibson

High Altitude Observatory, National Center for Atmospheric Research, Boulder, CO, USA

e-mail: tomczyk@ucar.edu
} 
Howard et al., 2006; Zhang et al., 2007; Temmer et al., 2010), and they are often associated with dramatic changes of coronal magnetic fields (e.g. Zhang and Low, 2005; Liu et al., 2009; Su and van Ballegooijen, 2012). Using mainly white-light coronagraphs, observations of CMEs are now made routinely both on the ground and in space. These instruments measure the polarized or total brightness of the corona, and CMEs are usually identified as large-scale disturbances in the coronal intensity-image sequences. White-light coronagraphs such as MK4 (Elmore et al., 2003; Reiner et al., 2003; Gibson et al., 2006) at the Mauna Loa Solar Observatory (MLSO) and the Large Angle Spectrometric Coronagraph (LASCO: Brueckner et al., 1995) onboard the Solar and Heliospheric Observatory (SOHO) have made great contributions to our understanding of the initiation and propagation of CMEs.

Spectroscopic observations of emission lines could provide valuable information on the plasma properties and dynamics in CMEs near the Sun (e.g. Harrison et al., 2003; Harra and Sterling, 2003; Ko et al., 2003; Lin et al., 2005; McIntosh, de Pontieu, and Leamon, 2010; Landi et al., 2010; Tian et al., 2012a; Giordano et al., 2013). However, conventional slit spectrographs such as the Extreme-Ultraviolet Imaging Spectrometer (EIS: Culhane et al., 2007) onboard Hinode and the Ultraviolet Coronagraph Spectrometer (UVCS: Kohl et al., 1995) onboard SOHO can only observe a small region. In addition, repeated raster scans of the same region can only be done at a low cadence (e.g. about five minutes for Hinode/EIS; see Tian et al., 2012a) because it takes minutes or even hours to scan the region. A filter instrument, on the other hand, can provide high-cadence observations of a large field of view in the solar corona, thus offering significant advantages over a spectrograph when observing large-scale solar eruptions such as CMEs. The LASCO-C1 instrument, which has revealed some important characteristics of CMEs' propagation in the low corona (e.g. Plunkett et al., 1997; Schwenn et al., 1997; Zhang et al., 2001), might be considered as one of such instruments. However, it took minutes to record a complete line profile (Mierla et al., 2005) so that the cadence could not be high. Moreover, this instrument only lasted for less than two years during solar minimum, making it not very useful for CME studies.

The Coronal Multichannel Polarimeter (CoMP: Tomczyk et al., 2008) is also such an instrument. It uses a narrow-band tunable filter to take high-cadence observations of the polarization state at a few spectral locations across the profiles of three infrared lines (Fe XIII $1074.7 \mathrm{~nm}$ and $1079.8 \mathrm{~nm}$, He I $1083.0 \mathrm{~nm}$ ). Images taken by CoMP have a field of view (FOV) of 1.05-1.40 solar radii, a spatial resolution of $4.46^{\prime \prime} \mathrm{pixel}^{-1}$, and a typical cadence of 30 seconds. The instrument was initially deployed at the National Solar Observatory/Sacramento Peak in 2004. Several successful observations of coronal Alfvén waves (Tomczyk et al., 2007; Tomczyk and McIntosh, 2009) and coronal cavities (Schmit et al., 2009; Dove et al., 2011) have been performed since then. The instrument was recently moved to the Mauna Loa Solar Observatory (MLSO) and started to obtain almost daily routine observations since October 2010.

Since CoMP can provide simultaneous measurements of the coronal line intensity, Doppler shift, line width, linear/circular polarization, and coronal density, it opens a completely new window for observations of the solar corona and CMEs. Here we report the first results of CoMP observations of CMEs. These observations might bring new insights into the initiation process of CMEs.

\section{Data Reduction and Correction}

Here we mainly use the three-point (sampled at three spectral locations $1074.50 \mathrm{~nm}$, $1074.62 \mathrm{~nm}, 1074.74 \mathrm{~nm}$ ) data of the Fe XIII $1074.7 \mathrm{~nm}$ line taken after December 2011. In 
these observations, sequences of the polarization (Stokes- $I,-Q$, and $-U$ only) images were obtained at each of these three spectral locations at a cadence of approximately 30 seconds. In this paper we mainly focus on the intensity (Stokes- $I$ ) data.

Although there are measurements at only three spectral locations, the intensity profile is in principle the same as the spectral line profile obtained by spectrographs. Thus, we can simply apply a least-squares single Gaussian fit to each intensity profile and obtain the line-center intensity, central wavelength, and line width (Tomczyk et al., 2007; Tomczyk and McIntosh, 2009). However, it takes too much time to apply the Gaussian fit to all intensity profiles in the full FOV for hundreds of frames. Fortunately, we found that a simple analytical solution can be derived from the three-point measurement. It is far more efficient to derive the line parameters by using the analytical solution.

If $I_{1}, I_{2}$, and $I_{3}$ are the measured intensities at known wavelengths (spectral locations) $\lambda_{1}, \lambda_{2}$, and $\lambda_{3}$, we have the following set of three equations:

$$
\begin{aligned}
& I_{1}=i \mathrm{e}^{\frac{-\left(\lambda_{1}-\lambda_{0}\right)^{2}}{w^{2}}}, \\
& I_{2}=i \mathrm{e}^{\frac{-\left(\lambda_{2}-\lambda_{0}\right)^{2}}{w^{2}}}, \\
& I_{3}=i \mathrm{e}^{\frac{-\left(\lambda_{3}-\lambda_{0}\right)^{2}}{w^{2}}} .
\end{aligned}
$$

It is clear that there are only three unknowns: the line center intensity [ $i]$, center wavelength $\left[\lambda_{0}\right]$, and line width $[w]$. Therefore, we should be able to directly derive these three unknowns from the three equations. If we take the natural logarithm of the ratios $I_{3} / I_{2}$ and $I_{1} / I_{2}$ and denote them as $a$ and $b$, we have

$$
\begin{aligned}
& a=\ln \left(\frac{I_{3}}{I_{2}}\right)=\frac{-\left(\lambda_{3}-\lambda_{0}\right)^{2}}{w^{2}}+\frac{\left(\lambda_{2}-\lambda_{0}\right)^{2}}{w^{2}}, \\
& b=\ln \left(\frac{I_{1}}{I_{2}}\right)=\frac{-\left(\lambda_{1}-\lambda_{0}\right)^{2}}{w^{2}}+\frac{\left(\lambda_{2}-\lambda_{0}\right)^{2}}{w^{2}} .
\end{aligned}
$$

If we take the sum $a+b$ and define $\delta$ as the spectral pixel size $\left(\lambda_{2}-\lambda_{1}\right.$ or $\left.\lambda_{3}-\lambda_{2}\right)$, the line width can be derived and expressed as

$$
w=\sqrt{\frac{-2 \delta}{a+b}} .
$$

The Doppler shift relative to $\lambda_{2}$ can be derived by taking the difference $a-b$ :

$$
v=\lambda_{0}-\lambda_{2}=\frac{w^{2}}{4 \delta}(a-b) .
$$

Finally, the line-center intensity can be computed as

$$
i=I_{2} \mathrm{e}^{\frac{v^{2}}{w^{2}}} .
$$

We show in Figure 1 three examples of observed intensity profiles and the line parameters derived by using the analytical solution. The solid line in each panel is the Gaussian function constructed by using the derived line parameters. Here positive and negative values of the Doppler shift mean red shift (away from the Earth) and blue shift (towards the Earth), 



Figure 1 Three examples of observed emission line profiles (diamonds) and the analytical solution (solid line). The dashed line indicates the rest wavelength of the line. The line center intensity [i], Doppler shift $[v]$, and line width $[w]$ are marked in each panel.

respectively. We do not remove the instrument filter width from the measured line width since the filter width has a fixed value, and we are only interested in changes of the line width.

Once we derive the line parameters for each intensity profile in the FOV, we can produce maps of intensity, Doppler shift, and line width. Maps generated from observations at different times can then be used to make movies of intensity, Doppler shift, and line width.

The Doppler-shift maps usually show predominant blue shift at the east limb and red shift at the west limb, which suggests an East-West trend in the line of sight (LOS) Doppler shift. This trend is at least partly caused by the rotation of the solar corona. We calculate the median value of Doppler shift at each solar- $x$ location to produce the East-West trend (Doppler shift as a function of solar- $x$ ). A median filter is then applied to this trend to eliminate possible abnormal values. Then we apply a fifth-order polynomial fit to the filtered trend. The resulting smooth trend is then subtracted from the map of Doppler shift.

Since there is no calibration lamp or cold lines, we could not perform an absolute wavelength calibration. We simply assume that the median value of Doppler shift is zero in each image. This assumption is usually valid since non-radial flows should on average cancel each other out (e.g. Hassler et al., 1999; Peter and Judge, 1999; Tian et al., 2010) at the limb and CoMP has such a large FOV. In addition, we are only interested in large Doppler shift perturbation which is unlikely to be affected by the accuracy of the absolute wavelength scale.

\section{First Results of CME Observations}

As we mentioned previously, CoMP provides simultaneous high-cadence (30 seconds) observations of coronal line intensity, Doppler shift, and line width in a large FOV for the first time. Such completely new types of observations may provide new insights into the processes of CME initiation and propagation. We have checked the CoMP data archive as well as the Atmospheric Imaging Assembly (AIA: Lemen et al., 2012) onboard the Solar Dynamics Observatory (SDO) and SOHO/LASCO data, and found 27 clear cases where CMEs or CME-related signatures were observed by CoMP between December 2011 and February 2013. Table 1 lists some information (observation date, approximate time when CoMP 
Table 1 CMEs observed by CoMP between December 2011 and February 2013.

\begin{tabular}{|c|c|c|c|c|c|c|}
\hline ID & Date & Time & $\begin{array}{l}\text { Flare } \\
\text { class }\end{array}$ & Limb & $\begin{array}{l}\text { White light } \\
\text { signature }\end{array}$ & Remarks \\
\hline 1 & 07 December 2011 & $20: 10$ & $\mathrm{C} 2.7$ & East & No & Loop oscillation \\
\hline 2 & 30 December 2011 & $20: 18$ & $\mathrm{C} 4.4$ & West & MK4 \& LASCO & $\begin{array}{l}\text { Flux rope, Type-II } \\
\text { burst }\end{array}$ \\
\hline 3 & 09 January 2012 & $20: 13$ & $\mathrm{C} 2.6$ & East & LASCO & \\
\hline 4 & 14 January 2012 & $21: 12$ & No & West & No & \\
\hline 5 & 15 March 2012 & $01: 33$ & $\mathrm{C} 1.1$ & West & MK4 \& LASCO & Prominence eruption \\
\hline 6 & 17 March 2012 & $23: 55$ & B8.1 & West & MK4 \& LASCO & \\
\hline 7 & 27 March 2012 & $21: 40$ & No & West & MK4 \& LASCO & \\
\hline 8 & 11 April 2012 & $20: 40$ & No & West & MK4 \& LASCO & \\
\hline 9 & 27 April 2012 & $17: 20$ & No & East & MK4 \& LASCO & \\
\hline 10 & 15 May 2012 & $17: 20$ & No & West & MK4 \& LASCO & Null reconnection \\
\hline 11 & 26 May 2012 & $20: 38$ & No & West & $\begin{array}{l}\text { Partial halo in } \\
\text { MK4 \& LASCO }\end{array}$ & $\begin{array}{l}\text { Originates from back } \\
\text { side of the Sun }\end{array}$ \\
\hline 12 & 01 June 2012 & $22: 16$ & C 3.3 & West & MK4 \& LASCO & Type-II burst \\
\hline 13 & 08 June 2012 & $23: 24$ & No & West & MK4 \& LASCO & Prominence eruption \\
\hline 14 & 06 July 2012 & 23:05 & $\mathrm{X} 1.1$ & West & MK4 \& LASCO & Type-II burst \\
\hline 15 & 08 July 2012 & 19:00 & No & West & MK4 \& LASCO & Slowly rising loops \\
\hline 16 & 13 July 2012 & $19: 45$ & $\mathrm{C} 1.3$ & West & No & \\
\hline 17 & 07 August 2012 & 19:02 & $\mathrm{C} 3.0$ & East & No & Prominence eruption \\
\hline 18 & 16 August 2012 & $00: 30$ & No & East & MK4 \& LASCO & Prominence eruption \\
\hline 19 & 24 August 2012 & $19: 30$ & No & East & LASCO & Narrow eruption \\
\hline 20 & 15 September 2012 & $22: 57$ & B9.6 & West & Faint in LASCO & Type-II burst \\
\hline 21 & 22 September 2012 & 19:30 & B9.2 & East & MK4 \& LASCO & \\
\hline 22 & 01 November 2012 & $21: 56$ & No & West & No & \\
\hline 23 & 12 November 2012 & $18: 55$ & $\mathrm{C} 2.0$ & East & LASCO & \\
\hline 24 & 08 January 2013 & 19:11 & $\mathrm{C} 4.0$ & West & No & Prominence eruption \\
\hline 25 & 01 February 2013 & $19: 23$ & No & West & LASCO & \\
\hline 26 & 04 February 2013 & $20: 43$ & $\mathrm{C} 1.9$ & East & LASCO & \\
\hline 27 & 15 February 2013 & 20:00 & No & East & LASCO & \\
\hline
\end{tabular}

observes the CME, associated flare class, east/west limb, white-light signatures, and other characteristics) of these CMEs. The data of flare class are taken from the Hinode Flare Catalogue (st4a.stelab.nagoya-u.ac.jp/hinode_flare/) and NOAA/SWPC Solar Region Summary (www.swpc.noaa.gov/ftpmenu/warehouse.html).

Inspection of the movies shows that the typical characteristics of CMEs in the CoMP data are the dramatic changes of Doppler shift and the obviously enhanced line width. Although all CMEs do show some perturbation in the image sequences of the intensity, the perturbation is much more obvious in the image sequences of the Doppler shift and line width. This is not difficult to understand since higher-order moments are usually more sensitive to changes. The significant changes of Doppler shift are probably largely associated with the coronal response to the mass eruptions and lateral expansion of CMEs. The increased line width might be caused by the enhanced flow inhomogeneity and turbulence in various substructures of CMEs in the LOS direction. 


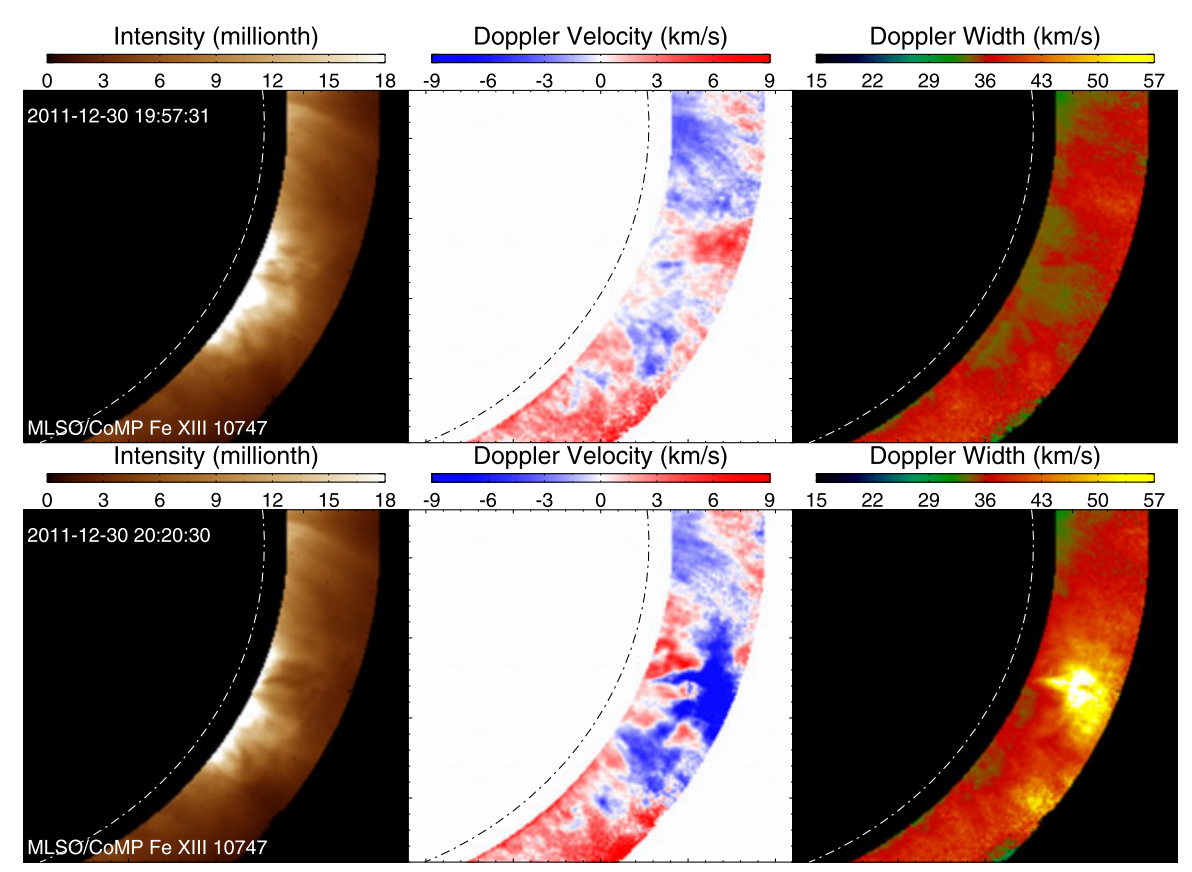

Figure 2 An eruption observed by CoMP on 30 December 2011. The first and second rows show images of the line center intensity, Doppler shift, and line width at 19:57:31 (before eruption) and 20:20:30 UT (during eruption), respectively. The dot-dashed line in each panel marks the limb of the solar disk. Two ESM movies (m2a.mov, m2b.mov) are associated with this figure.

We have to mention that the values of Doppler shift during CME eruption should not be interpreted as the line-of-sight component of the CME propagation speed in these observations. The reason is that the line wings are poorly sampled in our three-point measurements. However, the red and blue patterns in the Dopplergram do suggest that the plasma motions averaged along the line-of-sight direction are directed away and towards the observer, respectively. These motions include not only the outward movement of the CME, but also its lateral expansion and the dynamic response of the surrounding coronal plasma along the line-of-sight direction. This explains the complex Doppler-shift patterns in some CME eruption events. Nevertheless, the Doppler shift should experience a dramatic change in all CME eruption events and the largely perturbed (usually enhanced) Doppler shift should have an outward motion as the CME moves outward. Thus, the outward motion of largely enhanced Doppler shift is a good marker of CME eruption. In the future we plan to perform measurements of the line profile at more spectral locations. A better spectral sampling may help to separate these different types of motions due to their different speeds.

Figure 2 shows the three line parameters before and during the 30 December $2011 \mathrm{CME}$. The CME-caused changes of all line parameters can be clearly identified through a comparison between the first and second rows. Continuous evolution of different line parameters can be seen from the Electronic Supplementary Material (ESM) movies m2a.mov (full FOV) and m2b.mov (partial FOV). The outward propagating ejecta causes a dimming of $\approx 50 \%$ in the intensity, shifts the line center by about $20 \mathrm{~km} \mathrm{~s}^{-1}$ blueward, and enhances the line width by $\approx 20 \mathrm{~km} \mathrm{~s}^{-1}$. The spatial pattern of the intensity change roughly coincides with those of the Doppler-shift and line-width enhancement. 


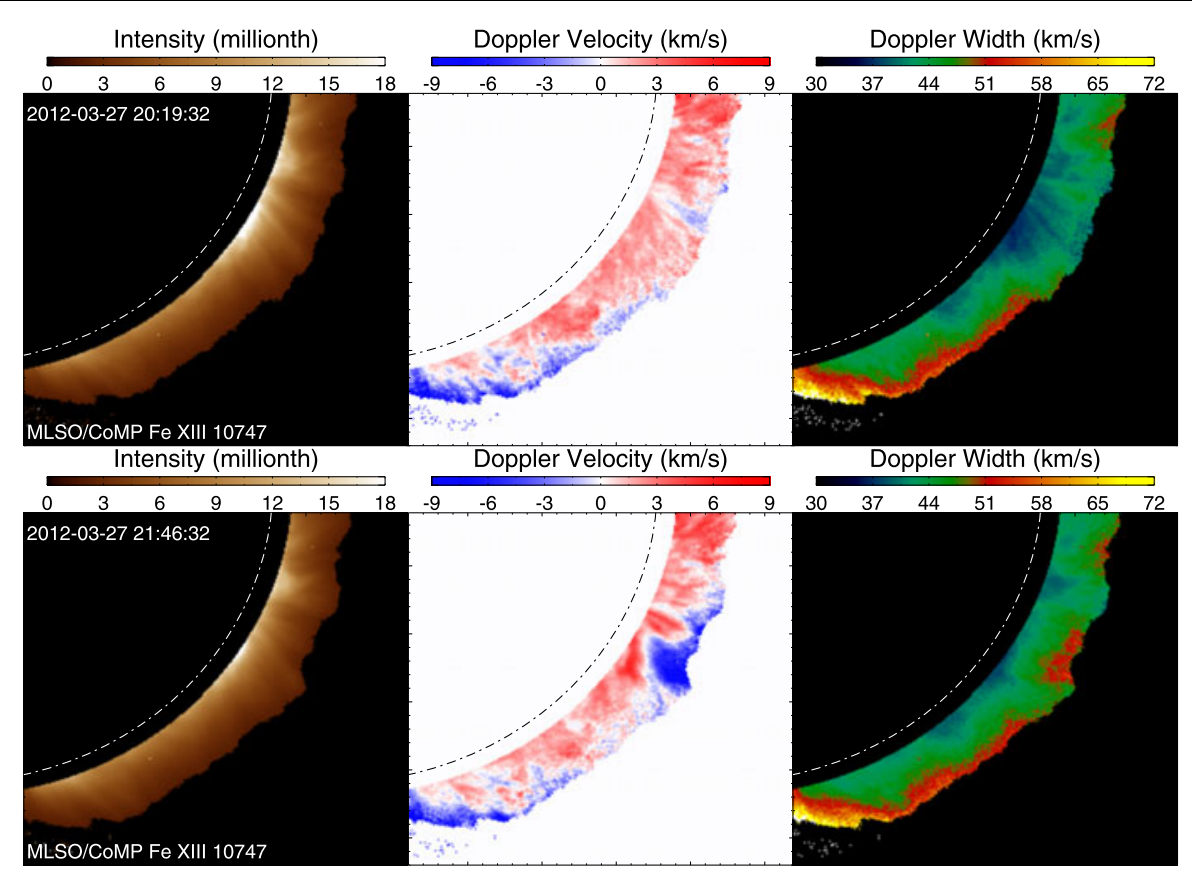

Figure 3 An eruption observed by CoMP on 27 March 2012. The first and second rows show images of the line-center intensity, Doppler shift, and line width at 20:19:32 (before eruption) and 21:46:32 UT (during eruption), respectively. The dot-dash line in each panel marks the limb of the solar disk. An ESM movie (m3.mov) is associated with this figure.

From LASCO movies (lasco-www.nrl.navy.mil/daily_mpg/), we can see that this CME developed into a ring-shaped propagating feature in the FOV of LASCO-C2 and -C3. Such a feature is likely to be a signature of flux rope or magnetic cloud (e.g., DeForest, Howard, and Tappin, 2011). From Figure 2 we can see that this CME erupts from an active region (AR). It is not clear whether the flux rope existed prior to eruption (e.g. Gibson et al., 2006) or formed during the eruption (e.g. Cheng et al., 2011).

A possible flux rope is also identified from the LASCO-C2 and -C3 movies on 13 October 2011. From AIA images, we can see that this flux-rope-type CME seems to originate from a coronal cavity at the northwest limb. Such a connection favors the flux-rope interpretation of coronal cavities (Low and Hundhausen, 1995; Gibson et al., 2006). Unfortunately, on that day CoMP data were only available prior to the CME eruption and the observation of the cavity is limited by an obstruction in the instrument. We also checked the list of cavities of Forland et al. (2011) but none of the erupting cavities were caught by CoMP. We hope that future observations may catch the complete process of flux-rope ejection and thus better our understanding of the role played by flux ropes in CME initiation and eruption.

The intensity perturbation of the 27 March 2012 CME is very small, which might be due to the fact that the CME propagates largely off the plane of sky (POS). Despite the weak signal in the intensity, we see an obvious change of the Doppler shift and line width. From Figure 3 and the ESM movie m3.mov we can clearly see the significant perturbation of Doppler shift and line width as the CME propagates into the FOV of CoMP.

The 7 December 2011 eruption revealed only very weak perturbation in the daily LASCO-C2 and -C3 movies. However, as we can see from Figures 4 and 5, this eruption was 


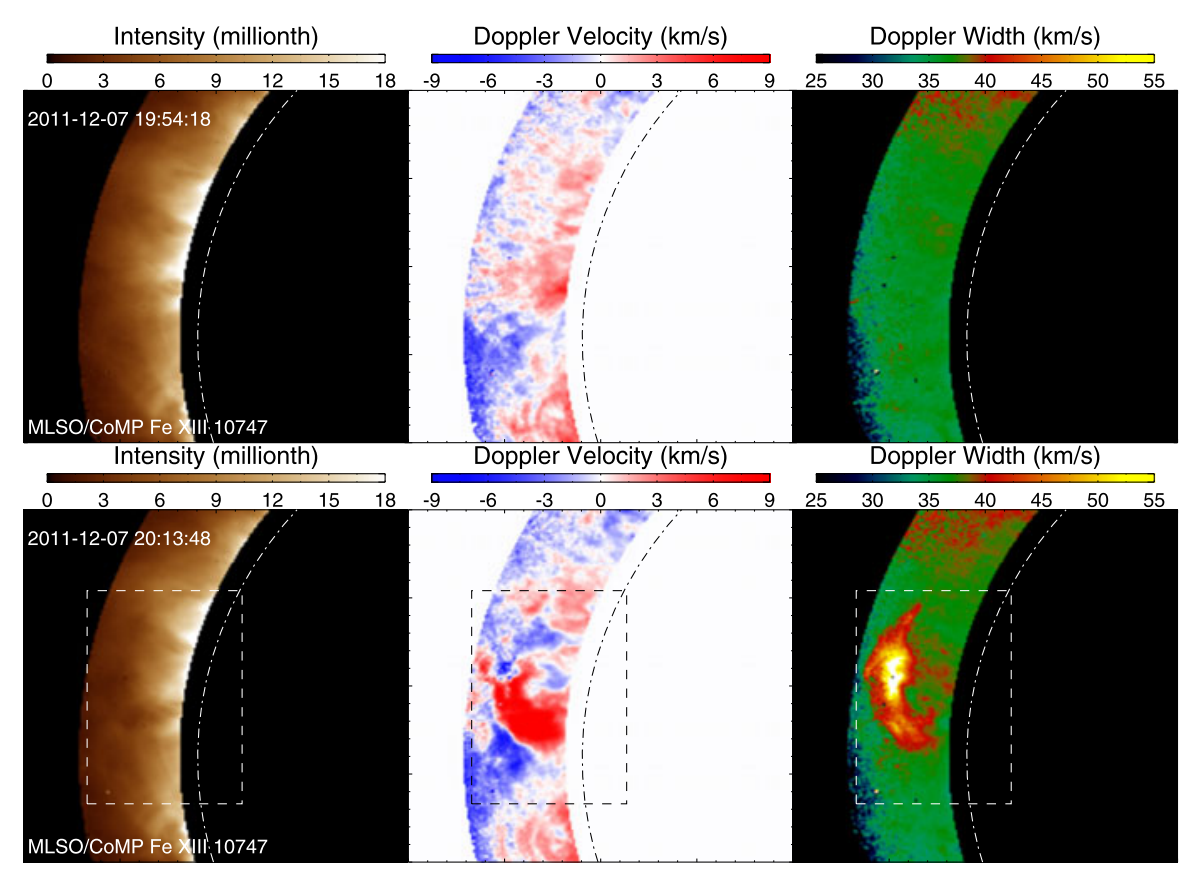

Figure 4 An eruption observed by CoMP on 7 December 2011. The first and second rows show images of the line-center intensity, Doppler shift, and line width at 19:54:18 and 20:13:48 UT, respectively. The dot-dashed line in each panel marks the limb of the solar disk. The rectangular region indicates the field of view shown in Figure 5. An ESM movie (m4.mov) is associated with this figure.

clearly recorded by both CoMP and SDO/AIA. The dominant ions in the $304 \AA$, $171 \AA$, and $193 \AA$ passbands are He II, Fe IX, and Fe XII, respectively (O'Dwyer et al., 2010). The eruption appears as a dark propagating feature in the CoMP intensity data, as can be seen from Figure 4 and the ESM movie m4.mov. The eruption also shifts the line center by about $20 \mathrm{~km} \mathrm{~s}^{-1}$ redward and enhances the line width by more than $20 \mathrm{~km} \mathrm{~s}^{-1}$. The dark feature roughly coincides with the enhanced line width and largely perturbed Doppler shift.

In Figure 5 we present the high-resolution AIA images in three passbands. These images were taken around 20:13:48 UT, the time when the images in the lower panel of Figure 5 were taken. The most enhanced line width (larger than $42 \mathrm{~km} \mathrm{~s}^{-1}$ ) region, which is also approximately the region where the most significant perturbation of the line center intensity and Doppler shift occurs, is outlined by the white contours in each panel. It seems that this region is immediately ahead of the ejecta. A comparison between Figures 4 and 5 suggests that the ejecta are also associated with large changes of Doppler shift and enhancement of the line width. However, these changes are clearly not as prominent as those ahead of the ejecta. The large perturbation ahead of the ejecta is likely largely caused by the large disturbance of the plasma and magnetic environment around the leading edge of the fast ejection.

Interestingly, we do not see any obvious dark propagating feature ahead of this ejecta in the AIA movies (not shown here). It is known that all of these three passbands of AIA have significant contribution from emission of cooler materials, whereas the CoMP emission is almost purely from the hot Fe XIII ion. But it is still not clear how the perturbation ahead of 


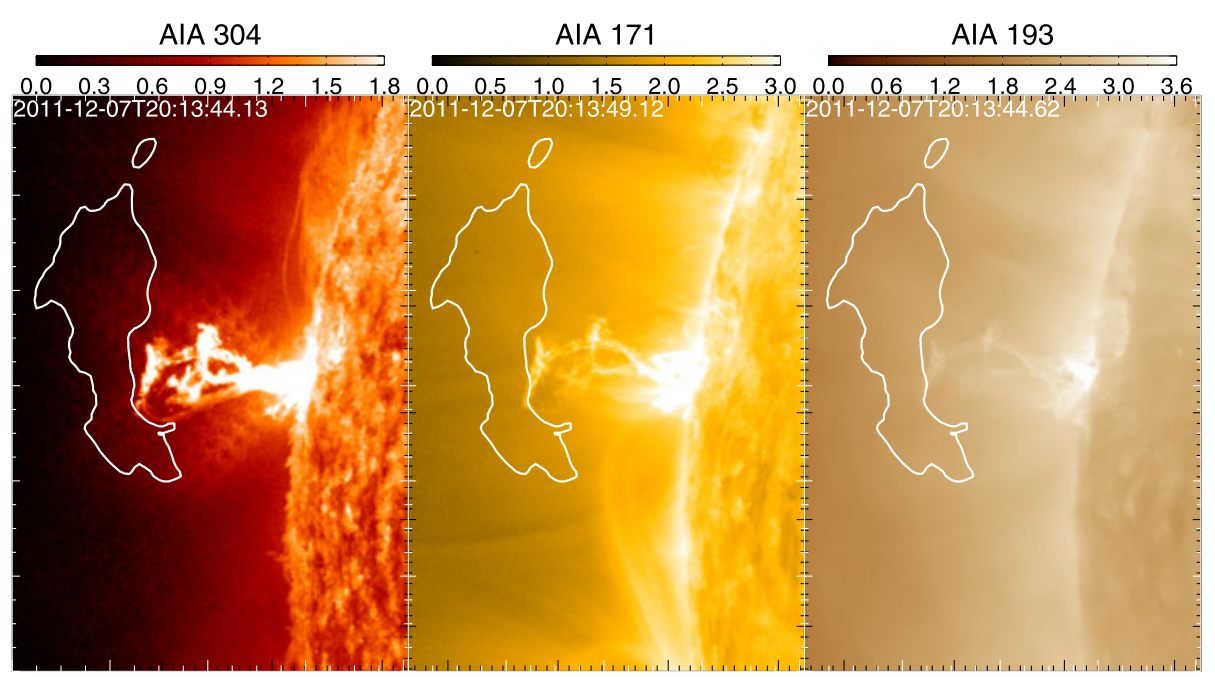

Figure 5 AIA observations of the 7 December 2011 event. Images of the AIA $304 \AA$, $171 \AA$, and $193 \AA$ passbands around 20:13:48 are presented from left to right. The contours mark locations where the line width of Fe XIII $1074.7 \mathrm{~nm}$ is larger than $42 \mathrm{~km} \mathrm{~s}^{-1}$.

the ejecta causes a reduction in the intensity of Fe XIII $1074.7 \mathrm{~nm}$ but no reduction in the AIA intensities.

Another interesting aspect of the 7 December 2011 eruption is the transverse oscillation of a loop system excited by the ejecta. The loop oscillation is best seen in the AIA $171 \AA$ movie (not shown here). The oscillating loop is clearly present in Figure 5 and is about $432 \mathrm{Mm}$ in length. The oscillation has a period of about 15 minutes and lasts for four cycles before damping out. The kink speed (twice the loop length divided by the period) at the apex of this oscillating loop can thus be calculated as $960 \mathrm{~km} \mathrm{~s}^{-1}$. The Alfvén speed inside the oscillating loop can be constrained to the range of $960 / \sqrt{2} \mathrm{~km} \mathrm{~s}^{-1}-960 \mathrm{~km} \mathrm{~s}^{-1}$ (e.g. Edwin and Roberts, 1983; Aschwanden et al., 1999; Nakariakov and Ofman, 2001; Wang and Solanki, 2004; Van Doorsselaere, Nakariakov, and Verwichte, 2008; Chen et al., 2011; Feng et al., 2011; Tian et al., 2012b; White and Verwichte, 2012). The two Fe XIII lines used by CoMP (1074.7 $\mathrm{nm}$ and $1079.8 \mathrm{~nm}$ ) can in principle be used to diagnose the coronal electron density. Combining information of the Alfvén speed and electron density, we can directly calculate the coronal magnetic-field strength. Unfortunately, this oscillating loop is barely resolved by CoMP. In addition, the density diagnostics of CoMP are still not finalized. We are planning to do joint observations of Hinode/EIS and CoMP, trying to use the density diagnostics of EIS to calibrate those of CoMP.

Figure 6 shows snapshots of CoMP observations of a partial-halo CME. A complete halo is seen at the viewpoint of STEREO-A. This eruption seems to originate from the backside of the Sun and propagates away from the Sun. The quickly developed large-scale disturbance can be clearly identified from movies of the Doppler shift and line width (available on mlso.hao.ucar.edu/mlso_datasum_comp.php?2012\&5\&26\&COMP, mainly at the west limb). Figure 6 clearly shows that the perturbation in the intensity data is not as significant as in the Doppler shift and line width, probably because of the strong foreground and background coronal emission. The quickly developed perturbation might be associated with the propagation of "EUV waves". Chen, Ding, and Chen (2010) suggests that spectroscopic observations may be used to identify the nature of "EUV waves", namely fast waves (e.g. Patsourakos and 


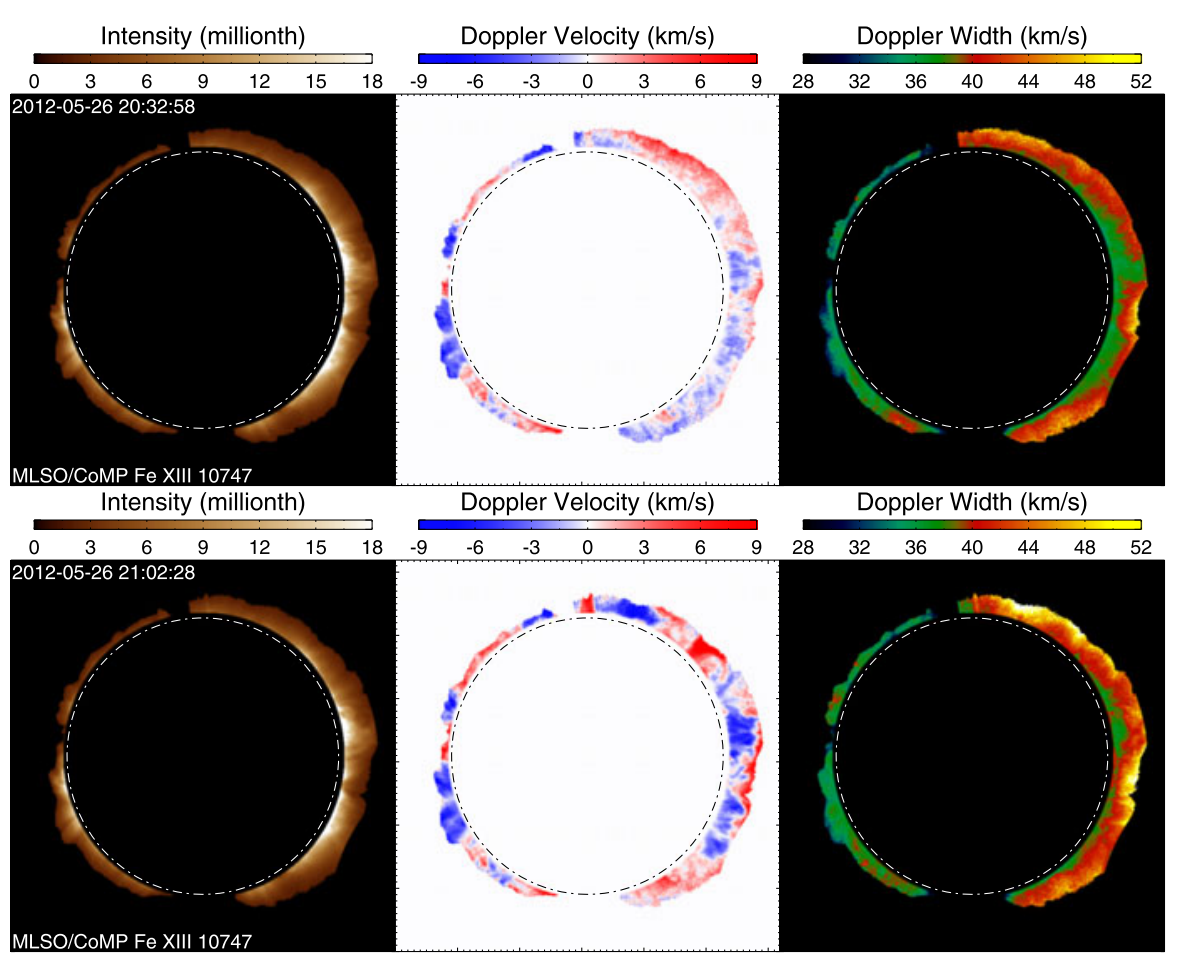

Figure 6 A partial halo-CME observed by CoMP on 26 May 2012. The first and second rows show images of the line-center intensity, Doppler shift and line width at 20:32:58 and 21:02:28 UT, respectively. The dot-dashed line in each panel marks the limb of the solar disk.

Vourlidas, 2009; Shen and Liu, 2012) or non-wave phenomenon such as successive stretching of magnetic field lines (e.g. Chen et al., 2002). Thus, combining numerical simulations and the spectroscopic-like large-FOV CoMP observations might reveal more insights into the process of "EUV waves".

CoMP observations of the 26 May 2012 CME suggest the importance of monitoring space weather on the ground. The 26 May 2012 CME first appears as a partial halo in the FOV of LASCO-C2 at 20:57, which is about 20 minutes later than the time (20:38 UT) when CoMP observes the larger-scale perturbation in the coronal line width and Doppler shift. So it is clear that CoMP observations can be very important for the prediction of Earth-directed halo CMEs. In this sense, observations of halo-CMEs by ground-based instruments such as CoMP provide a cost-effective and low-risk means of space weather monitoring.

\section{Summary and Future Perspectives}

CoMP provides high-cadence, large-FOV, spectroscopic-like observations of CMEs for the first time. We have presented first results of CME observations by CoMP in this article. Our results show that the primary characteristics of CMEs are the dramatic change of the Doppler shift and obviously enhanced line width. The information provided by these CoMP observations might be important for our better understanding of various CME processes such as the formation of flux ropes and propagation of "EUV waves". CoMP observations 
clearly demonstrate that space-weather monitoring is possible with inexpensive and lowrisk observations from the ground. So far we have identified 27 obvious CMEs in the CoMP data. With the increase of the solar activity, we expect to observe more CMEs in the future.

CoMP observations of CMEs are not restricted to the images of line-center intensity, Doppler shift, and line width. In fact CoMP measures the complete polarization state of three emission lines. The linear (Stokes- $Q$ and $-U$ ) and circular (Stokes- $V$ ) polarization data are not used in our analysis because our preliminarily processed polarization data shows no significant change of the linear polarization during CME eruptions and the circular-polarization signal is weak. We plan in the future to examine these data more thoroughly in order to establish whether changes may be evident if the data are averaged over longer time intervals, or whether a more optimal observation program might be established for analyzing CMEs with linear/circular polarization. Linear polarization is a promising diagnostic of coronal magnetic structures (e.g. Rachmeler et al., 2013) and it may be possible to demonstrate changes in magnetic topology by examining data before, during and after CME eruptions.

As we mentioned above, density diagnostics using the two Fe XIII lines is under way. Once this is finalized, we should be able to study density changes during CMEs, which could be used to estimate the CME mass (Harrison et al., 2003; Jin et al., 2009; Tian et al., 2012a). In addition, combining the density diagnostics and the Alfvén wave (Doppler shift oscillation) analysis (Tomczyk et al., 2007; Tomczyk and McIntosh, 2009; McIntosh, de Pontieu, and Tomczyk, 2008), we can produce maps of the electron density, Alfvén speed, and magnetic-field strength before and after CMEs. Such information can be used to constrain background coronal parameters prescribed in models of CMEs.

CoMP is a prototype of the Large-aperture Coronagraph component of the proposed COronal Solar Magnetism Observatory (COSMO). With a FOV of 1.05-2.0 solar radii, COSMO can greatly expand the ability of CME observations by CoMP. In addition, the Chromosphere and Prominence Magnetometer (ChroMag) component of COSMO can monitor solar activity on the disk. Thus, a combination of both components is likely to monitor the complete process of (halo) CME initiation and early phase propagation.

Acknowledgements SDO is the first mission of NASA's Living With a Star (LWS) Program. SDO data a courtesy of NASA/SDO and the AIA science team. H. Tian's work at CfA is supported under contract 8100002705 from Lockheed-Martin to SAO. Part of this work was done at NCAR, where H. Tian was supported under the ASP Postdoctoral Fellowship Program. The National Center for Atmospheric Research is sponsored by the National Science Foundation. We thank L. Sitongia for processing the CoMP level-0 data. We also thank P. Judge, B.C. Low, and H. Peter for helpful discussions.

Open Access This article is distributed under the terms of the Creative Commons Attribution License which permits any use, distribution, and reproduction in any medium, provided the original author(s) and the source are credited.

\section{References}

Aschwanden, M.J., Fletcher, L., Schrijver, C.J., Alexander, D.: 1999, Coronal loop oscillations observed with the Transition Region and Coronal Explorer. Astrophys. J. 520, 880 - 894. doi:10.1086/307502.

Brueckner, G.E., Howard, R.A., Koomen, M.J., Korendyke, C.M., Michels, D.J., Moses, J.D., Socker, D.G., Dere, K.P., Lamy, P.L., Llebaria, A., Bout, M.V., Schwenn, R., Simnett, G.M., Bedford, D.K., Eyles, C.J.: 1995, The Large Angle Spectroscopic Coronagraph (LASCO). Solar Phys. 162, 357 - 402. doi:10.1007/BF00733434.

Chen, F., Ding, M.D., Chen, P.F.: 2010, Spectroscopic analysis of an EIT wave/dimming observed by Hinode/EIS. Astrophys. J. 720, 1254 - 1261. doi:10.1088/0004-637X/720/2/1254.

Chen, P.F., Wu, S.T., Shibata, K., Fang, C.: 2002, Evidence of EIT and Moreton waves in numerical simulations. Astrophys. J. Lett. 572, L99-L102. doi:10.1086/341486. 
Chen, Y., Feng, S.W., Li, B., Song, H.Q., Xia, L.D., Kong, X.L., Li, X.: 2011, A coronal seismological study with streamer waves. Astrophys. J. 728, 147. doi:10.1088/0004-637X/728/2/147.

Cheng, X., Zhang, J., Liu, Y., Ding, M.D.: 2011, Observing flux rope formation during the impulsive phase of a solar eruption. Astrophys. J. Lett. 732, L25. doi:10.1088/2041-8205/732/2/L25.

Culhane, J.L., Harra, L.K., James, A.M., Al-Janabi, K., Bradley, L.J., Chaudry, R.A., Rees, K., Tandy, J.A., Thomas, P., Whillock, M.C.R., Winter, B., Doschek, G.A., Korendyke, C.M., Brown, C.M., Myers, S., Mariska, J., Seely, J., Lang, J., Kent, B.J., Shaughnessy, B.M., Young, P.R., Simnett, G.M., Castelli, C.M., Mahmoud, S., Mapson-Menard, H., Probyn, B.J., Thomas, R.J., Davila, J., Dere, K., Windt, D., Shea, J., Hagood, R., Moye, R., Hara, H., Watanabe, T., Matsuzaki, K., Kosugi, T., Hansteen, V., Wikstol, Ø.: 2007, The EUV Imaging Spectrometer for Hinode. Solar Phys. 243, 1961. doi:10.1007/s01007-007-0293-1.

DeForest, C.E., Howard, T.A., Tappin, S.J.: 2011, Observations of detailed structure in the solar wind at 1 AU with STEREO/HI-2. Astrophys. J. 738, 103. doi:10.1088/0004-637X/738/1/103.

Dove, J.B., Gibson, S.E., Rachmeler, L.A., Tomczyk, S., Judge, P.: 2011, A ring of polarized light: evidence for twisted coronal magnetism in cavities. Astrophys. J. Lett. 731, L1. doi:10.1088/2041-8205/ 731/1/L1.

Edwin, P.M., Roberts, B.: 1983, Wave propagation in a magnetic cylinder. Solar Phys. 88, $179-191$. doi:10.1007/BF00196186.

Elmore, D.F., Burkepile, J.T., Darnell, J.A., Lecinski, A.R., Stanger, A.L.: 2003, Calibration of a groundbased solar coronal polarimeter. In: Fineschi, S. (ed.) Soc. Photo-Optical Instrument. Eng. (SPIE) CS4843, 66 - 75. doi:10.1117/12.459279.

Feng, S.W., Chen, Y., Li, B., Song, H.Q., Kong, X.L., Xia, L.D., Feng, X.S.: 2011, Streamer wave events observed in solar cycle 23. Solar Phys. 272, 119 - 136. doi:10.1007/s11207-011-9814-6.

Forland, B., Rachmeler, L.A., Gibson, S.E., Dove, J.: 2011, Cavity magnetic observations: a survey using AIA and CoMP data. AGU Fall Meeting Abstracts, B1951.

Gibson, S.E., Foster, D., Burkepile, J., de Toma, G., Stanger, A.: 2006, The calm before the storm: the link between quiescent cavities and coronal mass ejections. Astrophys. J. 641, 590-605. doi:10.1086/500446.

Giordano, S., Ciaravella, A., Raymond, J., Ko, Y.K., Suleiman, R.: 2013, UVCS/SoHO catalog of coronal mass ejections from 1996 to 2005: spectroscopic proprieties. J. Geophys. Res. 118, 967-981. doi:10.1002/jgra.50166.

Gopalswamy, N., Lara, A., Yashiro, S., Kaiser, M.L., Howard, R.A.: 2001, Predicting the 1-AU arrival times of coronal mass ejections. J. Geophys. Res. 106, 29207-29218. doi:10.1029/2001JA000177.

Gosling, J.T., McComas, D.J., Phillips, J.L., Bame, S.J.: 1991, Geomagnetic activity associated with Earth passage of interplanetary shock disturbances and coronal mass ejections. J. Geophys. Res. 96, 7831 7839. doi:10.1029/91JA00316.

Harra, L.K., Sterling, A.C.: 2003, Imaging and spectroscopic investigations of a solar coronal wave: properties of the wave front and associated erupting material. Astrophys. J. 587, 429-438. doi:10.1086/ 368079.

Harrison, R.A., Bryans, P., Simnett, G.M., Lyons, M.: 2003, Coronal dimming and the coronal mass ejection onset. Astron. Astrophys. 400, 1071 - 1083. doi:10.1051/0004-6361:20030088.

Hassler, D.M., Dammasch, I.E., Lemaire, P., Brekke, P., Curdt, W., Mason, H.E., Vial, J.C., Wilhelm, K.: 1999, Solar wind outflow and the chromospheric magnetic network. Science 283, 810. doi:10.1126/science.283.5403.810.

Howard, T.A., Webb, D.F., Tappin, S.J., Mizuno, D.R., Johnston, J.C.: 2006, Tracking halo coronal mass ejections from 0-1 AU and space weather forecasting using the Solar Mass Ejection Imager (SMEI). J. Geophys. Res. 111, 4105. doi:10.1029/2005JA011349.

Jin, M., Ding, M.D., Chen, P.F., Fang, C., Imada, S.: 2009, Coronal mass ejection induced outflows observed with Hinode/EIS. Astrophys. J. 702, 27 - 38. doi:10.1088/0004-637X/702/1/27.

Ko, Y.K., Raymond, J.C., Lin, J., Lawrence, G., Li, J., Fludra, A.: 2003, Dynamical and physical properties of a post-coronal mass ejection current sheet. Astrophys. J. 594, 1068 - 1084. doi:10.1086/376982.

Kohl, J.L., Esser, R., Gardner, L.D., Habbal, S., Daigneau, P.S., Dennis, E.F., Nystrom, G.U., Panasyuk, A., Raymond, J.C., Smith, P.L., Strachan, L., van Ballegooijen, A.A., Noci, G., Fineschi, S., Romoli, M., Ciaravella, A., Modigliani, A., Huber, M.C.E., Antonucci, E., Benna, C., Giordano, S., Tondello, G., Nicolosi, P., Naletto, G., Pernechele, C., Spadaro, D., Poletto, G., Livi, S., von der Lühe, O., Geiss, J., Timothy, J.G., Gloeckler, G., Allegra, A., Basile, G., Brusa, R., Wood, B., Siegmund, O.H.W., Fowler, W., Fisher, R., Jhabvala, M.: 1995, The Ultraviolet Coronagraph Spectrometer for the Solar and Heliospheric Observatory. Solar Phys. 162, 313 - 356. doi:10.1007/BF00733433.

Landi, E., Raymond, J.C., Miralles, M.P., Hara, H.: 2010, Physical conditions in a coronal mass ejection from Hinode, Stereo, and SOHO observations. Astrophys. J. 711, 75-98. doi:10.1088/0004-637X/ 711/1/75. 
Lemen, J.R., Title, A.M., Akin, D.J., Boerner, P.F., Chou, C., Drake, J.F., Duncan, D.W., Edwards, C.G., Friedlaender, F.M., Heyman, G.F., Hurlburt, N.E., Katz, N.L., Kushner, G.D., Levay, M., Lindgren, R.W., Mathur, D.P., McFeaters, E.L., Mitchell, S., Rehse, R.A., Schrijver, C.J., Springer, L.A., Stern, R.A., Tarbell, T.D., Wuelser, J.P., Wolfson, C.J., Yanari, C., Bookbinder, J.A., Cheimets, P.N., Caldwell, D., Deluca, E.E., Gates, R., Golub, L., Park, S., Podgorski, W.A., Bush, R.I., Scherrer, P.H., Gummin, M.A., Smith, P., Auker, G., Jerram, P., Pool, P., Soufli, R., Windt, D.L., Beardsley, S., Clapp, M., Lang, J., Waltham, N.: 2012, The Atmospheric Imaging Assembly (AIA) on the Solar Dynamics Observatory (SDO). Solar Phys. 275, 17-40. doi:10.1007/s11207-011-9776-8.

Lin, J., Ko, Y.K., Sui, L., Raymond, J.C., Stenborg, G.A., Jiang, Y., Zhao, S., Mancuso, S.: 2005, Direct observations of the magnetic reconnection site of an eruption on 2003 November 18. Astrophys. J. 622, 1251 - 1264. doi:10.1086/428110.

Liu, Y., Luhmann, J.G., Lin, R.P., Bale, S.D., Vourlidas, A., Petrie, G.J.D.: 2009, Coronal mass ejections and global coronal magnetic field reconfiguration. Astrophys. J. Lett. 698, L51-L55. doi:10.1088/0004-637X/698/1/L51.

Low, B.C., Hundhausen, J.R.: 1995, Magnetostatic structures of the solar corona. 2: The magnetic topology of quiescent prominences. Astrophys. J. 443, $818-836$. doi:10.1086/175572.

McIntosh, S.W., de Pontieu, B., Tomczyk, S.: 2008, A coherence-based approach for tracking waves in the solar corona. Solar Phys. 252, 321 - 348. doi:10.1007/s11207-008-9257-x.

McIntosh, S.W., de Pontieu, B., Leamon, R.J.: 2010, The impact of new EUV diagnostics on CME-related kinematics. Solar Phys. 265, 5 - 17. doi:10.1007/s11207-010-9538-z.

Mierla, M., Schwenn, R., Teriaca, L., Stenborg, G., Podlipnik, B.: 2005, Using LASCO-C1 spectroscopy for coronal diagnostics. Adv. Space Res. 35, 2199-2203. doi:10.1016/j.asr.2005.04.031.

Nakariakov, V.M., Ofman, L.: 2001, Determination of the coronal magnetic field by coronal loop oscillations. Astron. Astrophys. 372, L53 - L56. doi:10.1051/0004-6361:20010607.

O’Dwyer, B., Del Zanna, G., Mason, H.E., Weber, M.A., Tripathi, D.: 2010, SDO/AIA response to coronal hole, quiet Sun, active region, and flare plasma. Astron. Astrophys. 521, A21. doi:10.1051/0004-6361/ 201014872.

Patsourakos, S., Vourlidas, A.: 2009, "Extreme ultraviolet waves" are waves: first quadrature observations of an extreme ultraviolet wave from STEREO. Astrophys. J. Lett. 700, L182-L186. doi:10.1088/0004-637X/700/2/L182.

Peter, H., Judge, P.G.: 1999, On the Doppler shifts of solar ultraviolet emission lines. Astrophys. J. 522, 1148 - 1166. doi:10.1086/307672.

Plunkett, S.P., Brueckner, G.E., Dere, K.P., Howard, R.A., Koomen, M.J., Korendyke, C.M., Michels, D.J., Moses, J.D., Moulton, N.E., Paswaters, S.E., St. Cyr, O.C., Socker, D.G., Wang, D., Simnett, G.M., Bedford, D.K., Biesecker, D.A., Eyles, C.J., Tappin, S.J., Schwenn, R., Lamy, P.L., Llebaria, A.: 1997, The relationship of green-line transients to white-light coronal mass ejections. Solar Phys. 175, 699718. doi:10.1023/A:1004981125702.

Rachmeler, L.A., Gibson, S.E., Dove, J.B., DeVore, C.R., Fan, Y.: 2013, Polarimetric properties of flux ropes and sheared arcades in coronal prominence cavities. Solar Phys. accepted.

Reiner, M.J., Vourlidas, A., Cyr, O.C.S., Burkepile, J.T., Howard, R.A., Kaiser, M.L., Prestage, N.P., Bougeret, J.L.: 2003, Constraints on coronal mass ejection dynamics from simultaneous radio and whitelight observations. Astrophys. J. 590, 533-546. doi:10.1086/374917.

Schmit, D.J., Gibson, S.E., Tomczyk, S., Reeves, K.K., Sterling, A.C., Brooks, D.H., Williams, D.R., Tripathi, D.: 2009, Large-scale flows in prominence cavities. Astrophys. J. Lett. 700, L96-L98. doi:10.1088/0004-637X/700/2/L96.

Schwenn, R., Inhester, B., Plunkett, S.P., Epple, A., Podlipnik, B., Bedford, D.K., Eyles, C.J., Simnett, G.M., Tappin, S.J., Bout, M.V., Lamy, P.L., Llebaria, A., Brueckner, G.E., Dere, K.P., Howard, R.A., Koomen, M.J., Korendyke, C.M., Michels, D.J., Moses, J.D., Moulton, N.E., Paswaters, S.E., Socker, D.G., St. Cyr, O.C., Wang, D.: 1997, First view of the extended green-line emission corona at solar activity minimum using the LASCO-C1 coronagraph on SOHO. Solar Phys. 175, 667-684. doi:10.1023/A:1004948913883.

Shen, Y., Liu, Y.: 2012, Evidence for the wave nature of an extreme ultraviolet wave observed by the Atmospheric Imaging Assembly on board the Solar Dynamics Observatory. Astrophys. J. 754, 7. doi:10.1088/0004-637X/754/1/7.

Su, Y., van Ballegooijen, A.: 2012, Observations and magnetic field modeling of a solar polar crown prominence. Astrophys. J. 757, 168. doi:10.1088/0004-637X/757/2/168.

Temmer, M., Veronig, A.M., Kontar, E.P., Krucker, S., Vršnak, B.: 2010, Combined STEREO/RHESSI study of coronal mass ejection acceleration and particle acceleration in solar flares. Astrophys. J. 712, 14101420. doi:10.1088/0004-637X/712/2/1410.

Tian, H., Tu, C., Marsch, E., He, J., Kamio, S.: 2010, The nascent fast solar wind observed by the EUV imaging spectrometer on board Hinode. Astrophys. J. Lett. 709, L88-L93. doi:10.1088/2041-8205/ 709/1/L88. 
Tian, H., McIntosh, S.W., Xia, L., He, J., Wang, X.: 2012a, What can we learn about solar coronal mass ejections, coronal dimmings, and extreme-ultraviolet jets through spectroscopic observations? Astrophys. $J$. 748, 106. doi:10.1088/0004-637X/748/2/106.

Tian, H., McIntosh, S.W., Wang, T., Ofman, L., De Pontieu, B., Innes, D.E., Peter, H.: 2012b, Persistent Doppler shift oscillations observed with Hinode/EIS in the solar corona: spectroscopic signatures of Alfvénic waves and recurring upflows. Astrophys. J. 759, 144. doi:10.1088/0004-637X/759/2/144.

Tomczyk, S., McIntosh, S.W.: 2009, Time-distance seismology of the solar corona with CoMP. Astrophys. J. 697, 1384 - 1391. doi:10.1088/0004-637X/697/2/1384.

Tomczyk, S., McIntosh, S.W., Keil, S.L., Judge, P.G., Schad, T., Seeley, D.H., Edmondson, J.: 2007, Alfvén waves in the solar corona. Science 317, 1192. doi:10.1126/science.1143304.

Tomczyk, S., Card, G.L., Darnell, T., Elmore, D.F., Lull, R., Nelson, P.G., Streander, K.V., Burkepile, J., Casini, R., Judge, P.G.: 2008, An instrument to measure coronal emission line polarization. Solar Phys. 247, 411 - 428. doi:10.1007/s11207-007-9103-6.

Van Doorsselaere, T., Nakariakov, V.M., Verwichte, E.: 2008, Detection of waves in the solar corona: kink or Alfvén? Astrophys. J. Lett. 676, L73 - L75. doi:10.1086/587029.

Wang, T.J., Solanki, S.K.: 2004, Vertical oscillations of a coronal loop observed by TRACE. Astron. Astrophys. 421, L33 - L36. doi:10.1051/0004-6361:20040186.

Wang, Y.M., Ye, P.Z., Wang, S., Zhou, G.P., Wang, J.X.: 2002, A statistical study on the geoeffectiveness of Earth-directed coronal mass ejections from March 1997 to December 2000. J. Geophys. Res. 107, 1340. doi:10.1029/2002JA009244.

White, R.S., Verwichte, E.: 2012, Transverse coronal loop oscillations seen in unprecedented detail by AIA/SDO. Astron. Astrophys. 537, A49. doi:10.1051/0004-6361/201118093.

Zhang, M., Low, B.C.: 2005, The hydromagnetic nature of solar coronal mass ejections. Annu. Rev. Astron. Astrophys. 43, 103 - 137. doi:10.1146/annurev.astro.43.072103.150602.

Zhang, J., Dere, K.P., Howard, R.A., Kundu, M.R., White, S.M.: 2001, On the temporal relationship between coronal mass ejections and flares. Astrophys. J. 559, 452 - 462. doi:10.1086/322405.

Zhang, J., Richardson, I.G., Webb, D.F., Gopalswamy, N., Huttunen, E., Kasper, J.C., Nitta, N.V., Poomvises, W., Thompson, B.J., Wu, C.C., Yashiro, S., Zhukov, A.N.: 2007, Solar and interplanetary sources of major geomagnetic storms (Dst $=-100 \mathrm{nT}$ ) during 1996-2005. J. Geophys. Res. 112(11), 10102. doi:10.1029/2007JA012321. 